\title{
RELATIVE SIZE IN ISOLATION AS A STIMULUS FOR RELATIVE PERCEIVED DISTANCE ${ }^{1}$
}

\author{
WILLIAM EPSTEIN AND STEPHEN S. BARATZ \\ University of Kansas
}

\begin{abstract}
2 experiments examined the perceived relative distance produced by relative size in isolation. The hypothesis was that $\mathrm{S}$ assumes identity of physical size and interprets the difference in apparent size as a difference in distance. Experiment I examined this hypothesis by observing the effect of experimentally trained size assumptions on subsequent relative distance judgments. Experiment II used pairs of familiar objects which had known, normally invariant physical sizes. By varying the actual physical sizes of the standards it was possible to study the hypothesized effect of size assumptions on relative perceived distance. The results of Experiment I did not agree with the hypothesis. Experiment II did confirm the hypothesis. Both experiments were discussed in the context of the assumptive hypothesis.
\end{abstract}

Relative size is a stimulus correlate of perceived relative distance. The object which subtends the smaller visual angle appears more distant. This is the case when all other stimulus correlates, e.g., motion perspective, are eliminated, as well as under unrestricted conditions of viewing. One explanation of the effects of relative size under restricted conditions of observation is suggested in the following definition of the relative-size cue:

For a given ratio of visual angles, assuming the ratio of physical sizes determines the ratio of distances. If the assumed sizes are equal, the object subtending the larger visual angle appears nearer and the one subtending the small appears farther [Ittelson, 1960, p. 69].

This definition proposes that the effect of relative size is mediated by the subjective assumption of identity. Ames (1946) has expressed this view succintly: "You presume identity of size ... from similarity, and use the appearance of difference (in size) as an indication of distance [p. 46]." Two experiments were conducted to examine this hypothesis.

1 This work was supported by Research Grant MH 04153-03 made to the first author by the National Institute of Mental Health of the United States Public Health Service.

\section{EXPERIMENT I}

\section{Method}

General.-The first stage consisted of a training procedure designed to establish strong associations between the color of a target and its physical size. The intention was to train $S$ to assume the size of the target from observation of its color. In the second stage, pairs of equidistant colored targets were presented under reduced conditions of observation, and $S$ reported their relative distance. The size and color of the targets were systematically varied in a manner designed to reveal the effect of the earlier training on the judgments of distance.

Apparatus.-A modified version of the table used in earlier experiments (Epstein, $1961,1963)$ was the main apparatus. This apparatus allows the standard and comparison objects to be presented in separate fields. The standard field can be viewed only monocularly, while simultaneously the comparison field is viewed binocularly. The standard alley contained two light boxes whose centers were $28 \mathrm{~cm}$. above the table top (approximate eye level) and separated laterally by $22.5 \mathrm{~cm}$. forming an angle of $10^{\circ}$ with $S$ as the vertex. The faces of the boxes were in $S$ 's frontal plane at a distance of $150 \mathrm{~cm}$. from the viewing aperture. The standard squares were inserted in holders in the faces of the boxes. Under the experimental conditions, only the standard squares were visible in the standard alley. The comparison alley was well lighted and contained two tracks which formed an angle of $10^{\circ}$ with $S$ as the vertex. Riding on each track was a cart which carried an ir- 
TABLE 1

Size-Color Conjunctions Learned BY the EXPERIMENTAL $S$ s

\begin{tabular}{l|c|c|c}
\hline \hline \multirow{2}{*}{$\begin{array}{c}\text { Color of } \\
\text { Training }\end{array}$} & \multicolumn{2}{|l}{ Size (In.) of Square during Training } \\
\cline { 2 - 4 } & $\begin{array}{c}\text { Subgroup } \\
1\end{array}$ & $\begin{array}{c}\text { Subgroup } \\
2\end{array}$ & $\begin{array}{c}\text { Subgroup } \\
\mathbf{3}\end{array}$ \\
\hline Blue, yellow & 4 & 2 & 1 \\
Red, brown & 2 & $\mathbf{1}$ & 4 \\
Orange, green & 1 & 4 & 2 \\
\hline
\end{tabular}

regularly contoured nonsense form. The two forms were equal in area but were differently shaped. By pulling two strings $S$ was able to vary the distance of the comparison forms. During the experiment $S$ 's head was restrained in a chin-rest head-clamp arrangement to discourage movement.

Materials.-The standards were 1-, 2-, and 4-in. squares of layers of colored tissue paper mounted in black cardboard frames. Six colors were used: yellow, blue, brown, red, orange, and green.

Subjects.-Forty-two students from an introductory psychology course served as $S \mathrm{~s}$, 30 of whom were in the experimental group while 12 were control $S$ s. The assignment to conditions was in a random order.

Procedure.-In the first stage the experimental $S$ s performed an oddity task. On each of 48 trials a set of three squares differing in size and/or color was presented, and $S$ was instructed to point to the square "which does not belong with the others." The conditions of the task were contrived to encourage $S$ to learn the prescribed size-color conjunctions. Objective evidence that $S$ learned these conjunctions was provided by a test of free recall following the last trial. There were three subgroups of $10 \mathrm{Ss}$. Each group learned a different set of color-size conjunctions. The plan is shown in Table 1. By combining the data for these subgroups any effects of color per se on apparent distance (Johns \& Summer, 1948; Over, 1962) would be distributed evenly among the various comparisons.

Immediately following the training task $S$ began the second stage. This consisted of a series of judgments of the relative distances of the members of 20 pairs of squares. The 20 pairings can be classified into three categories: (1) Nine trials on which both members of the pair were from the same trained size category but differed in physical size when presented. (2) Six trials on which members of the pair were from different trained size categories but were the same physical size when presented. (3) Five trials on which the members of the pair were from different size categories and also differed in physical size when presented. Table 2 contains a schematic description of illustrative trials in each classification.

The $S$ was instructed to report his immediate impression of the relative distance of the squares. The main part of the instructions was as follows:

I will designate a square by calling out its color and I would like you to move the cart which is on the same side as the square (left or right) to a position along the track which is the same distance from you as the square. Then I will ask you to set the second nonsense form so that it is at the same distance in relation to the first form as is the second colored square to the first colored square.

Each $S$ made one ascending and one descending judgment of the distance of each of the members of each pair. On half the trials $S$ matched the right-hand square first and on the other trials he matched the left-hand square first. The position (left or right) of the members of each pair was varied between $S$ s. The 20 pairings were presented in random order.

At the conclusion of the distance judgments each experimental $S$ was tested for the retention of the color-size associations which he learned in the preliminary training. The $S$ was shown a series of six black cardboard squares which duplicated the dimensions of the standards, and he was asked to designate the color which was associated with each size during the training.

The procedure for the control $S$ s was the same except that the first stage was omitted.

\section{Results}

Inspection of the data did not reveal any systematic effect of direction of judgment (ascending or descending) or order of judgment (left or right square matched first) on the apparent radial separation of the standards. Therefore, the results for these conditions were combined. Table 3 shows the mean apparent distances of the standards for the experimental and control $S \mathrm{~s}$ on each of the eight trials shown in Table 2. The results for the 
TABLE 2

Schematic Description of a Sample of Trials and the Theoretical Expectations

\begin{tabular}{c|c|c|c|c|c|c}
\hline \multirow{2}{*}{ Class } & \multicolumn{2}{|c|}{ Left Square } & \multicolumn{3}{c}{ Right Square } & \multicolumn{2}{c}{ Predictions $^{b}$} \\
\cline { 2 - 6 } & Size Traineds & Size Presented & Size Trained & Size Presented & Assumptional & Visual Angle \\
\hline 1 & L & L & L & I & RF & RF \\
1 & I & S & I & L & RN & RN \\
2 & L & I & I & L & RN & E \\
2 & L & I & I & I & RN & E \\
2 & L & S & I & S & E & RF \\
3 & L & L & I & I & E & RN \\
3 & S & S & I & I & RN & LN \\
3 & L & L & S & I & RN \\
\hline
\end{tabular}

a $\mathrm{L}=$ large, $\mathrm{I}=$ intermediate, $\mathrm{S}=$ small.

$\mathrm{b}$ RF = right square farther; $\mathrm{RN}=$ right square nearer; $\mathrm{LN}=$ left square nearer; $\mathrm{E}=$ squares equidistant.

remaining 12 trials are not given. ${ }^{2}$ In every case the results for the trials which are omitted were consistent with the data for the reported trials in their respective classes.

The predictions in the two righthand columns of Table 2 have been labeled assumptional and visual angle. The assumptional predictions are those derived from the theoretical account of relative size described in the

${ }^{2}$ Extended versions of Tables 2-5 which include the data for all the trials have been deposited with the American Documentation Institute. Order Document No. 7861 from ADI Auxiliary Publications Project, Photoduplication Service, Library of Congress, Washington, D. C. 20540. Remit in advance $\$ 1.25$ for microfilm or $\$ 1.25$ for photocopies and make checks payable to: Chief, Photoduplication Service, Library of Congress. introduction. The visual-angle predictions are made entirely on the basis of relative visual angle, disregarding the presumed influence of assumptions. Introducing assumptional considerations on Class 1 trials does not lead to predictions which differ from those based on visual-angle relationships alone. However, on the trials in Classes 2 and 3 the assumptional hypothesis leads to predictions which differ from those derived solely by taking into account the difference in visual angle. A comparison between the expectations presented in Table 2 and the data in Table 3 shows that in every instance the outcome was predictable entirely on the basis of relative visual angle.

TABLE 3

Mean Apparent Distances of Standards for the Trials Described in Table 2

\begin{tabular}{c|c|c|c|c|c|c}
\hline & \multicolumn{2}{|c|}{ Experimental $S s(N=30)$} & \multicolumn{3}{c}{ Control $S_{s}(N=12)$} \\
\cline { 2 - 6 } Class & Left Square & Right Square & $\begin{array}{c}\text { Apparent Radia) } \\
\text { Separation }\end{array}$ & Left Square & Right Square & $\begin{array}{c}\text { Apparent Radial } \\
\text { Separation }\end{array}$ \\
\hline 1 & 133 & 153 & 20 & 114 & 161 & 47 \\
1 & 185 & 128 & 57 & 197 & 127 & 70 \\
2 & 136 & 137 & 1 & 122 & 128 & 6 \\
2 & 154 & 153 & 1 & 156 & 156 & 6 \\
2 & 183 & 183 & 28 & 183 & 189 & 6 \\
3 & 131 & 159 & 28 & 116 & 159 & 43 \\
3 & 185 & 157 & 28 & 189 & 149 & 40 \\
3 & 130 & 156 & 26 & 122 & 160 & 38 \\
\hline
\end{tabular}


Table 3 also shows that the outcomes for the experimental and control Ss were similar. The only effect of the pretraining seems to have been a reduction in the magnitude of the relative size effect for the experimental $S$ s. The mean difference in the perceived distance of the standards was smaller for the experimental $S$ s on 17 of the 20 trials. The difference between the overall mean separations for the 20 trials for the two groups was significant, $t(18)=5.64, \quad p<.01$. The diminution of the difference in perceived distance cannot be construed as evidence of the influence of assumptions. In fact on the trials in Class 2 the diminution contradicts the assumptional expectations. In general, the smaller separations which were obtained after the pretraining were not related to the assumptional thesis in any systematic manner.

The test of retention of the trained size-color conjunctions yielded unequivocal results. All $S$ s exhibited complete retention.

\section{EXPERIMENT II}

Experiment I tried to establish specific assumptions experimentally by training $S$ with the standards. It is possible, however, that this procedure failed to achieve its purpose. The training period was relatively brief, and may have been insufficient for the purpose of establishing a stable, compelling color-size entailment. It is true that $S$ s exhibited perfect retention of the size-color conjunctions; however, the possibility remains that these conjunctions were not sufficiently overlearned to become spontaneously operative during the distance judgments. In addition, the color of an object is not normally a reliable prognostic indicator of size. This may have vitiated the effects of the training procedure. For these reasons an experiment was conducted to examine the influence of assumptions which $S$ may be presumed to have developed through extended and frequent everyday commerce with the standards.

\section{Method}

Apparatus.-The apparatus was the same as in Exp. I except that the light boxes used to display the standards were removed. In Exp. II the standards were mounted at a distance of $135 \mathrm{~cm}$. and illuminated by two focused beams of light which were restricted to the surfaces of the standards.

Materials.-There were nine standards, each a photograph of a dime, quarter, or halfdollar. Each coin was represented in its normal size, and also in the sizes of the two other coins; e.g., the quarter was reduced to the size of the dime and enlarged to the size of the half-dollar. The diameters of a normal dime, quarter, and half-dollar are 1.7,2.5, and $3.0 \mathrm{~cm}$., respectively.

Subjects. - Thirty-two new students from the same undergraduate population which served in Exp. I were the $S$ s.

Procedure.-Relative-distance judgments of 23 pairs of standards were obtained following the procedure of Exp. I. The pairings may be classified in the same way as in Exp. I. There were 9 pairings in Class 1, 9 in Class 2, and 5 in Class 3. A sample of the pairings is represented in Table 4. The letters $H, Q$, and D represent the half-dollar, quarter, and dime photographs, respectively. The three sizes, large (normal, half-dollar size), intermediate (normal, quarter size) and small (normal, dime size) are represented by the letters L, I, and S, respectively. The 23 pairings were presented to each $S$ in one of four different random orders. In addition, the following three variables were counterbalanced: temporal order of ascending and descending judgments; lateral position of the standard judged first; and the lateral position of each standard.

\section{Results}

Table 4 records the two predictions for each trial. The pairings in Classes 2 and 3 are of special interest since the introduction of assumptional considerations leads to expectations which differ from those dictated by visual- 
angle relationships. As an example Pairing $3 \mathrm{c}$ in Table 4 may be examined. In terms of relative visual angle HL should appear closer. However, if $S$ assumes that the relative physical sizes of $H$ and $D$ correspond to their real-life relative sizes, then the larger than usual apparent size of $D$ relative to $H$ will cause DI to appear nearer than HL. For convenience, the data in Table 4 have been recorded in two columns labeled Left and Right. The means in the Left column represent the apparent distances of the left-hand members of the pairs listed in the Pairing column and the means in the Right column represent the apparent distances of the righthand members. Thus for Pairing 1a DS appeared at $176.71 \mathrm{~cm}$. and DI at $125.62 \mathrm{~cm}$. Since the position of each coin was varied between $S \mathrm{~s}$, the designations left and right in Table 4 do not refer to the location of the standard.

The results shown in Table 4 are as unequivocal as those obtained in Exp. I. However, whereas Exp. I failed to provide evidence for the influence of assumptions, the results for all seven critical trials in Classes 2 and 3 of Table 4 support the assumptional hy- pothesis. This was also true for the remaining seven trials in Classes 2 and 3.

A supplementary analysis provided additional support for the assumptional interpretation. If the difference in the perceived distance between standards is mediated by assumptive factors, then the magnitude of the difference should be determined similarly. As an illustration, Pairing 2b may be compared with Pairing $2 a$ (see Table 4). In both cases the assumptional hypothesis predicts separation while visual-angle considerations suggest equidistance. However, on closer consideration it will be apparent that the assumptional view also demands that the difference in perceived distance for Pairing $2 \mathrm{~b}$ be greater than for Pairing $2 \mathrm{a}$. This follows, in the most general way, from the fact that the discrepancy between assumed and actual physical sizes is greater for Pairing 2b. Table 5 shows a sample of the 12 comparisons which were made. In order to make these comparisons more convincing we have compared trials which presented the same visual-angle relationships. Table 5 shows that the results for all the comparisons confirmed the require-

TABLE 4

Sample of Pairings Presented in Exp. II : Theoretically Required and Obtained Relative Distance

\begin{tabular}{|c|c|c|c|c|c|c|}
\hline \multirow{2}{*}{ Class } & \multirow{2}{*}{ Pairing } & \multicolumn{2}{|c|}{ Predictions } & \multicolumn{2}{|c|}{ Obtained Apparent Distance } & \multirow{2}{*}{$t$} \\
\hline & & Assumptional & Visual Angle & Left & Right & \\
\hline $1 \mathrm{a}$ & DS vs. DI & DI nearer & same & 176.21 & 125.62 & $10.56^{* *}$ \\
\hline $1 \mathrm{~b}$ & DI vs. DL & $\mathrm{DL}$ nearer & same & 148.18 & 119.56 & $7.22 * *$ \\
\hline $2 \mathrm{a}$ & DS vs. QS & D nearer & equidistant & 150.53 & 193.84 & $7.95^{* *}$ \\
\hline $2 \mathrm{~b}$ & DS vs. HS & D nearer & equidistant & 151.12 & 213.34 & $9.48^{* *}$ \\
\hline $2 \mathrm{c}$ & DI vs. QI & $\mathrm{D}$ nearer & equidistant & 127.96 & 158.15 & $5.90^{* *}$ \\
\hline $2 \mathrm{~d}$ & DI vs. HI & $\mathrm{D}$ nearer & equidistant & 130.59 & 177.40 & $6.14^{* *}$ \\
\hline $3 a$ & QI vs. DS & equidistant & $Q$ nearer & 147.71 & 154.03 & 0.35 \\
\hline $3 b$ & $\mathrm{HL}$, vs. QI & equidistant & H nearer & 152.43 & 157.68 & 1.18 \\
\hline $3 c$ & HL vs. $\widetilde{D} I$ & $\mathrm{D}$ nearer & H nearer & 159.56 & 133.21 & $3.39^{* *}$ \\
\hline
\end{tabular}

Note.-Letter subscripts in class designations are used to distinguish pairings within the same class. $* * p<.01$ 
TABLE 5

Predicted and Obtained Apparent Radial SEPARATIONS FOR COMPARISONS BETWEEN PAIRINGS

\begin{tabular}{c|c|c|c|c}
\hline \hline \multirow{2}{*}{$\begin{array}{c}\text { Predicted Radial } \\
\text { Separations } \\
\text { for Selected } \\
\text { Comparisons }\end{array}$} & \multicolumn{3}{|c}{ Obtained Radial Separations } \\
\cline { 2 - 5 } & Pairing & $\begin{array}{c}\text { Sepa- } \\
\text { ration }\end{array}$ & Pairing & $\begin{array}{c}\text { Sepa- } \\
\text { ration }\end{array}$ \\
\hline $1 \mathrm{a}>3 \mathrm{a}$ & $1 \mathrm{a}$ & 51.09 & $3 \mathrm{a}$ & 6.32 \\
$1 \mathrm{~b}>3 \mathrm{~b}$ & $1 \mathrm{~b}$ & 28.62 & $3 \mathrm{~b}$ & 5.25 \\
$2 \mathrm{~b}>2 \mathrm{a}$ & $2 \mathrm{~b}$ & 62.22 & $2 \mathrm{a}$ & 43.31 \\
$2 \mathrm{~b}>2 \mathrm{c}$ & $2 \mathrm{~b}$ & 62.22 & $2 \mathrm{c}$ & 30.19 \\
$2 \mathrm{~d}>2 \mathrm{c}$ & $2 \mathrm{~d}$ & 46.81 & $2 \mathrm{c}$ & 30.19 \\
\hline
\end{tabular}

ments of the assumptional hypothesis. The same was true for the remaining seven comparisons.

Especially convincing are two special cases not included in Table 5 . These are comparisons between Pairing $Q I$ vs. QL and Pairing DI vs. HL, and between Pairing $\mathrm{HS}$ vs. $\mathrm{HI}$ and Pairing DS vs. HI. In these cases the assumptional hypothesis predicts a difference in the direction of apparent separation despite the identical retinal stimulation. The standards subtending the larger visual angles should appear nearer when both the members of the pair have the same identity. In contrast the standard subtending the smaller visual angle should appear nearer when the members of the pair are different coins. These expectations were confirmed.

\section{Discussion}

The two experiments produced conflicting results. It might be argued that the pretraining task in Exp. I failed to modify the long established assumptions which $S$ brought with him into the experimental situation. Nevertheless, in so far as the present findings are concerned, any positive conclusions about the role of assumptions must be restricted to the case of representative objects of known size. In this connection it is important to note that the relative per- ceived distance cannot be predicted by treating each standard as if it had been presented alone. If the formula derived by Epstein (1963, p. 261) from the known size-apparent distance hypothesis (Epstein, Park, \& Casey, 1961, pp. 503-507) is applied to each member of the standard pair the outcome does not agree with the judged distances obtained in the present experiment. This occurs despite the fact that the formula provides remarkably accurate predictions for the case when a single standard is presented (Epstein, 1963). Evidently the simultaneous presence of two standards affects the perceived distance of each.

If the pretraining in Exp. I actually did modify $S$ 's assumptions about size identity, then there remains the original problem of accounting for the consistent unidirectional results which are usually obtained with nonrepresentational standards. Alternative resolutions are not hard to formulate; however, they do not appear promising. As an example, consider the minimum principle (Hochberg, 1957) which has been successfully applied to other instances of depth perception in which $S$ shows a preference for one of several alternative perceptual outcomes all of which are equivalent in terms of the retinal stimulation (e.g., Hochberg \& Brooks, 1960). This is the principle that the perceptual system decodes the stimulus imput in accordance with the requirements of informational economy. More specific to the present problem, the minimum principle implies that if several alternative percepts are possible, then the percept which requires the least amount of information to specify will have the highest probability of occurrence. In order to apply this principle a ranking is needed of the alternatives in terms of the information which is required to specify each one. It is difficult to see the basis for assigning ranks in the present experiments.

We should note, however, that the hypothesis of Ittelson and Ames is also not without difficulties. These authors assert that $S$ must, on a conscious or subconscious level, make the assumption 
of identity in order that the reported perceptual experience occur. This view depends ultimately for its validity on evidence of the functioning of assumptions independent of the perceptual response which is presumed to be the consequence of these assumptions. This evidence is not available. In the absence of such data all that one can say is that $S$ responds as if he were making the assumption of identity. In this formulation the hypothesis is considered to be explanatory but not necessarily descriptive of the psychological processes which mediate the effect. Therefore, its validity will depend on successful prediction, rather than demonstrations of the reality status of the identity assumption.

\section{REFERENCES}

Ames, A., JR. Some demonstrations concerned with the origin and nature of our sensations. Hanover, N. H.: Institute for Associated Research, 1946.
Epstein, W. The known size apparent distance hypothesis. Amer. J. Psychol., $1961,74,333-346$.

Epstein, W. The influence of assumed size on apparent distance. Amer. J. Psychol., 1963, 76, 257-265.

Epstein, W., Park, J., \& Casery, A. The current status of the size-distance hypotheses. Psychol. Bull., 1961, 58, 491-514.

HOCHBERG, J. Effect of the Gestalt revolution: The Cornell symposium on perception. Psychol. Rev., 1957, 64, 73-84.

HOCHBERG, J., \& BROoKs, V. The psychophysics of form: Reversible perspective drawings of spatial objects. Amer. $J$. Psychol., 1960, 73, 337-354.

ItTELson, W. H. Visual space perception. New York: Springer, 1960.

Johns, E. H., \& Summer, F. C. Relation of the brightness differences of colors to their apparent distances. J. Psychol., 1948, 26, 25-29.

OVER, R. Stimulus wavelength variation and size and distance judgments. Brit. $J$. Psychol., 1962, 53, 141-148.

(Received June 21, 1963) 